\title{
Extracorporeal Membrane Oxygenation for 67 Days as a Bridge to Heart Transplantation in a Postcardiotomy Patient with Failing Heart and Mediastinitis
}

\author{
Hyoung Woo Chang, M.D., Yang Hyun Cho, M.D., Ph.D., Suhyun Cho, R.N., Kiick Sung, M.D., Ph.D., \\ and Pyo Won Park, M.D., Ph.D.
}

Department of Thoracic and Cardiovascular Surgery, Samsung Medical Center, Sungkyunkwan University School of Medicine, Seoul, Korea

\begin{abstract}
We report a case of successful heart transplantation after 67 days of support with venoarterial extracorporeal membrane oxygenation (ECMO) in a patient who underwent surgery for type A aortic dissection and myocardial infarction complicated by irreversible myocardial damage and a deep sternal wound infection. During ECMO support, left heart vent and distal limb perfusion were performed. Mediastinitis was treated with mediastinal washout and irrigation. Multiple complications from peripheral ECMO were successfully managed.
\end{abstract}

Key Words: cardiomyopathy; extracorporeal membrane oxygenation; heart; mediastinitis; shock; transplantation.

Currently, a ventricular assist device (VAD) is the preferred method of mechanical circulatory support as a bridge to heart transplantation.[1] However, in patients with both a failing heart and mediastinal infection, placing a cannula or pump inside the mediastinum might be contraindicated. There have been reports of peripherally-cannulated venoarterial (VA) extracorporeal membrane oxygenation (ECMO) support as a bridge to heart transplantation.[2,3] If durable mechanical support is contraindicated, patients with intractable heart failure depending on VA ECMO frequently die from ECMO-related complications before receiving heart transplantation.[4]

\section{Case Report}

A 44-year-old female $(170 \mathrm{~cm}, 65 \mathrm{~kg})$ with a family history of Marfan syndrome presented to another institution with severe chest pain and circulatory shock. She had cardiac arrest during coronary angiography, which revealed type A aortic dissection and obstruction of the left main coronary ostium. After

Received on April 3, 2015 Revised on May 20, 2015

Accepted on October 12, 2015

Correspondence to: Pyo Won Park, Department of Thoracic and Cardiovascular Surgery, Samsung Medical Center, Sungkyunkwan University School of Medicine,

81 Irwon-ro, Gangnam-gu, Seoul 06351, Korea

Tel: +82-2-3410-1677, Fax: +82-2-3410-0089

E-mail: pwpark@skku.edu

${ }^{*}$ No potential conflict of interest relevant to this article was reported. establishment of VA ECMO through femoral vessels, she had emergent ascending aorta and root replacement along with coronary artery bypass surgery. Despite surgery, she was kept on VA ECMO because of extensive myocardial damage. After the initial operation, she underwent procedures to control bleeding three times. The sternum was kept open and only the skin was approximated. On ECMO day \#15, she was trans- 
ferred to our institution in order to be evaluated for heart transplantation.

On ECMO day \#16, we brought her to the operating room to close the sternum in order to reduce her level of sedation. We found that the mediastinal space was filled with pus. Tissue adhesion was extensive, and collateral vessels had developed. Irrigation tubes were placed in the mediastinum, which was irrigated for six sequential days. Vancomycin was used because methicillin-resistant Staphylococcus aureus was cultured from the mediastinal fluid. On ECMO day \#19, a left ventricular vent cannula was inserted via right anterior thoracotomy due to progression of pulmonary edema

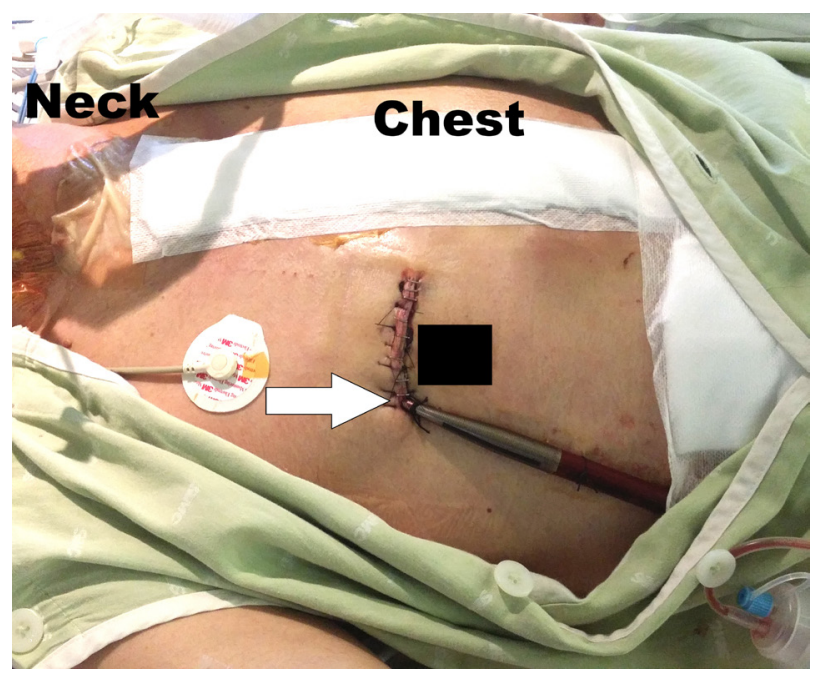

Fig. 1. Left ventricular vent catheter inserted via right anterior thoracotomy (arrow).
(Fig. 1 and Fig. 2). Initially, percutaneous trans-septal left atrial venting was considered. However, the right femoral vein was not available because it was being used for venous cannulation, and the left femoral vein was not suitable for vent cannula insertion because arterial cannula was in the left femoral artery. After discussion with cardiologists, we chose to insert the vent cannula directly into the right upper pulmonary vein via anterior thoracotomy.

The patient's status fluctuated. We maintained ECMO flow to keep central venous oxygen saturation above $70 \%$. Coagulation, bleeding, and hemolysis were monitored by activated clotting time, plasma hemoglobin, and lactate dehydrogenase, in addition to other labs. Heparin was our primary anticoagulant, but argatroban was used when we suspected heparin-induced thrombocytopenia. After percutaneous tracheostomy, we were able to initiate rehabilitation. During the peak bleeding period, she required transfusion of two or three packed red blood cells daily for more than a week (139 units of packed red blood cells were transfused during the ECMO support period). The patient was lightly sedated, but not paralyzed. During ECMO support in our institution, the circuit (both oxygenator and pump head) was replaced three times, and the pump head was exchanged three times.

There were two notable catheter-related events. On ECMO day \#32, the arterial cannula in the left femoral artery was found to be partially withdrawn. Because there was severe bleeding, we immediately placed another 16 Fr arterial can-
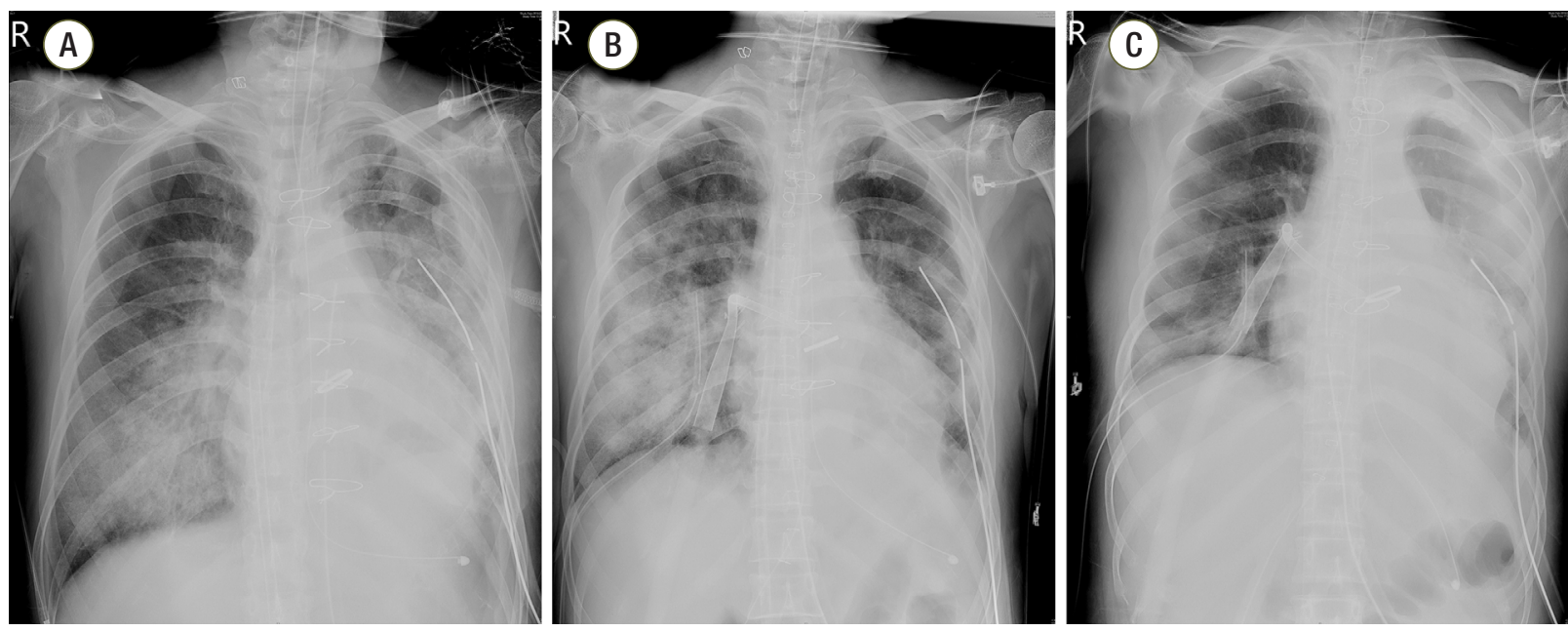

Fig. 2. Chest radiographs that were obtained before (A), immediately after (B), and two days after (C) vent cannula insertion. Pulmonary edema had resolved in $(\mathrm{C})$. 


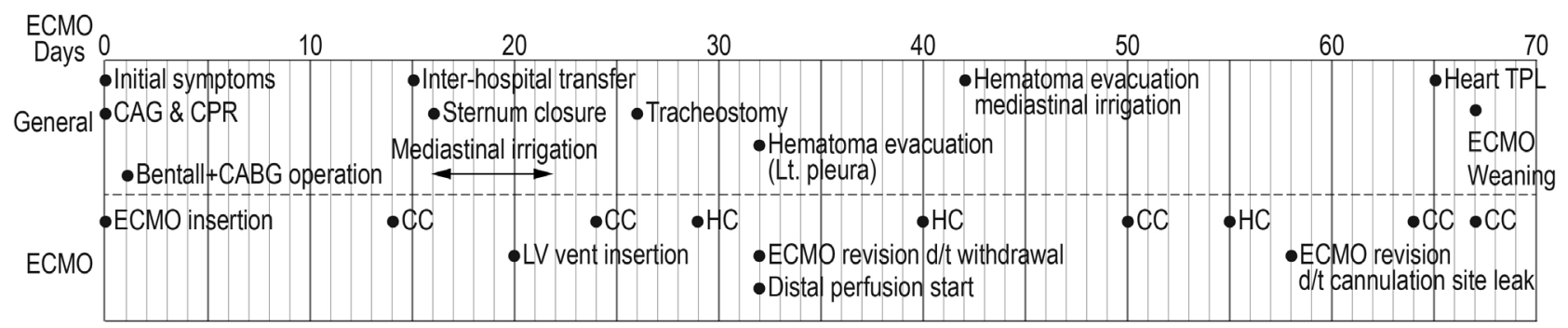

Fig. 3. Summary of key events during ECMO support. ECMO: extracorporeal membrane oxygenator; CAG: coronary angiography; CPR: cardiopulmonary resuscitation; CABG: coronary artery bypass grafting; TPL: transplantation; CC: circuit (oxygenator \& pump) change; HC: head (centrifugal pump) change; LV: left ventricle.

nula (Edwards Lifesciences, Irvine, CA, USA) in the right femoral artery. A 7 Fr arterial introducer (Terumo, Shibuya, Japan) was also inserted for distal perfusion. On ECMO day \#64, the arterial cannula in the right femoral artery was replaced with a new cannula of larger diameter (18 Fr) due to intractable blood leak. Key events during ECMO support are summarized in Fig. 3.

On ECMO day \#65, orthotopic heart transplantation was performed. After confirmation of clean mediastinum, a donor heart was procured. The cold and warm ischemic times were 179 and 87 minutes, respectively. Because the recipient was already on ECMO support, the heart was well decompressed and she was stable during cardiectomy. However, we failed to wean the patient from ECMO on the same day due to intractable bleeding and suboptimal oxygen saturation. Two days later (ECMO day \#67), her vital signs were stable and bleeding stopped. She was smoothly weaned from ECMO. The duration of ECMO support was 67 days, and the patient was discharged home on the 110th day from first resuscitation with ECMO. The patient had no neurologic sequelae. We have been following the patient for 6 months as an outpatient, and her functional class is New York Heart Association class I.

\section{Discussion}

Peripherally-cannulated VA ECMO support for more than a month is rare for several reasons, including death on ECMO and conversion to long-term mechanical support.[4,5] When this patient was transferred to our institution, our initial goal was to perform heart transplantation. However, her mediastinitis was a contraindication to heart transplantation. Moreover, weaning from ECMO was not possible, as her cardiac function did not improve. We were unable to switch from ECMO to VAD, because the mediastinal space was infected. Although it was a complicated situation, we believed we could treat the deep sternal wound infection and proceed to heart transplantation, which we were able to achieve in this patient. In fact, bleeding and mediastinal infection are not uncommon complications for a postcardiotomy ECMO patient. Although many surgeons may think heart transplantation in a patient with deep sternal wound infection is unrealistic, proper management of the sternal wound often clears the infection.

Because placing a cannula in the mediastinum was contraindicated, we had to use peripheral cannulae. This resulted in bleeding around the femoral cannulation site during ECMO support. Although the bleeding was successfully managed by meticulous anticoagulation, skin sutures, and cannula change, prolonged use of peripheral cannulation was certainly an issue. Frequent discontinuation of heparin also resulted in the need for pump and oxygenator changes. In summary, we managed a patient with massive myocardial infarction and deep sternal wound infection after surgery for type A aortic dissection. Although those conditions were significant challenges, we successfully bridged the patient to heart transplantation.

\section{ORCID}

Hyoung Woo Chang http://orcid.org/0000-0001-5516-0333 


\section{References}

1) Francis GS, Greenberg BH, Hsu DT, Jaski BE, Jessup M, LeWinter MM, et al: ACCF/AHA/ACP/HFSA/ ISHLT 2010 clinical competence statement on management of patients with advanced heart failure and cardiac transplant: a report of the ACCF/AHA/ACP Task Force on Clinical Competence and Training. J Am Coll Cardiol 2010; 56: 424-53.

2) Barth E, Durand M, Heylbroeck C, Rossi-Blancher M, Boignard A, Vanzetto G, et al: Extracorporeal life support as a bridge to high-urgency heart transplantation. Clin Transplant 2012; 26: 484-8.
3) Cho YH, Yang JH, Sung K, Jeong DS, Park PW, Kim WS, et al: Extracorporeal life support as a bridge to heart transplantation: importance of organ failure in recipient selection. ASAIO J 2015; 61: 139-43.

4) Aubron C, Cheng A, Pilcher D, Leong T, Magrin G, Cooper DJ, et al: Factors associated with outcomes of patients on extracorporeal membrane oxygenation support: a 5-year cohort study. Crit Care 2013; 17: R73.

5) Guttendorf J, Boujoukos AJ, Ren D, Rosenzweig MQ, Hravnak M: Discharge outcome in adults treated with extracorporeal membrane oxygenation. Am J Crit Care 2014; 23: 365-77. 